Pacific Journal of Mathematic 


\title{
THE ALGEBRA OF BOUNDED CONTINUOUS FUNCTIONS INTO A NONARCHIMEDEAN FIELD
}

\author{
RICHARD STAUM
}

\begin{abstract}
Let $S$ be a topological space, $F$ a complete nonarchimedean rank 1 valued field, and $C^{*}(S, F)$ the Banach algebra of bounded, continuous, $F$-valued functions on $S$. Various topological conditions on $S$ and/or $F$ are shown to be equivalent, respectively, to each of the following: every maximal ideal of $C^{*}(S, F)$ is fixed; the only quotient field of $C^{*}(S, F)$ is $F$ itself; every homomorphism of $C^{*}(S, F)$ into $F$ is an evaluation at a point of $S$; the Stone-Weierstrass theorem holds for $C^{*}(S, F)$. It is also shown that a certain topological space derived from $S$ may be embedded in the space of maximal ideals of $C^{*}(S, F)$ with Gelfand topology, or in the space of homomorphisms of $C^{*}(S, F)$ into $F$.
\end{abstract}

O. Introduction. Throughout this paper, $C^{*}(S, F)$ denotes the Banach algebra of bounded, continuous functions on a topological space $S$ into a complete nonarchimedean rank 1 valued field $F$. We introduce several stronger-than-usual topological separation properties, such as ultrahausdorff, ultraregular, and ultranormal; and several weaker-than-usual compactness properties, such as mildly compact, mildly countably compact, and mildly Lindelof. We then show that several key implications involving $C^{*}(S, F)$ become equivalences when the new topological properties replace their conventional counterparts.

In $\S 1$, we define and discuss these new topological properties, and relate them to the cofilters ("ouf-filtres") of van der Put [13]. In $\S 2$, we obtain a result on the metric structure of non-locally compact nonarchimedean Banach spaces.

In $\S 3$, we show that all maximal ideals of $C^{*}(S, F)$ are fixed if and only if $S$ is mildly compact (Theorem 15); and that $F$ is the only quotient field of $C^{*}(S, F)$ if and only if $F$ is locally compact or $S$ is mildly countably compact (Theorem 19 ). Using the result of $\S 2$, we also give necessary and/or sufficient conditions for the only homomorphisms of $C^{*}(S, F)$ into $F$ to be evaluations at points of $S$ (Theorems 20 and 21). We also show that the set of quasicomponents of $S$, appropriately topologized, is homeomorphic to the space of fixed maximal ideals of $C^{*}(S, F)$, with either of the Gelfand topologies defined by Shilkret [14] (Theorems 10 and 12).

In $\S 4$, we extend results of Kaplansky [7] and Chernoff, Rasala, and Waterhouse [3]: we introduce two versions of the Stone-Weierstrass property, and show that the stronger version in $C^{*}(S, F)$ is equivalent to mild compactness of $S$, and the weaker version is sufficient for mild 
countable compactness of $S$ (Theorems 22 and 23).

It is interesting to note that many of our results involve properties of $S$ only, and are independent of the choice of the nonarchimedean field $F$.

1. Topology, A quasicomponent of $S$ is a minimal nonempty intersection of sets clopen in $S$. The quasicomponents form a partition of $S$ into closed sets. Each quasicomponent is a union of components; if $S$ is compact and Hausdorff, the quasicomponents and components are identical [6].

Distinct points or sets in $S$ will be called ultraseparated if they are contained in disjoint clopen sets. $S$ will be called ultrahausdorff, or UT2, if distinct points are ultraseparated; equivalently, if every quasicomponent is a singleton. After Ellis [4], $S$ will be called ultraregular, or $U R$, if disjoints points and closed sets are ultraseparated; equivalently, if $S$ has a basis consisting of clopen sets. $S$ will be called ultranormal, or $U N$, if disjoint closed sets are ultraseparated.

$S$ is totally disconnected, or $T D$, if every component is a singleton. Hence, if $S$ is ultrahausdorff, it is totally disconnected; and if $S$ is compact, Hausdorff, and totally disconnected, then it is ultrahausdorff. We also note that, for a $T_{1}$ space, ultranormality implies ultraregularity, and ultraregularity implies the ultrachausdorff property. For a compact space, the ultrahausdorff property implies ultraregularity, and ultraregularity implies ultranormality.

LEMMA 1. In an ultraregular space, every open or closed set is a union of quasicomponents.

Proof. If $S$ is ultraregular, then every open set is a union of clopen sets and hence a union of quasicomponents. It follows that every closed set, being the complement of an open set, is also a union of quasicomponents.

LEMMA 2. Let $G$ be a family of functions on a set $A$ into a topological space $B$, and let $A$ be topologized with the weak-G topology. Then:

(1) If $B$ is ultraregular, $A$ is ultraregular.

(2) If $B$ is ultrahausdorff, and $G$ separates points of $A$, then $A$ is ultrahausdorff.

Proof. (1) If $B$ is ultraregular, it has a clopen basis. The preimages, under the members of $G$, of these clopen sets form a clopen subbasis for $A$. Hence $A$ is ultraregular. 
(2) Let $p$ and $q$ be distinct points of $A$. If $G$ separates points, then $g(p) \neq g(q)$ for some $g$ in $G$. If $B$ is ultrahausdorff, then $g(p)$ and $g(q)$ are contained in disjoint clopen sets $V$ and $W$ of $B$. Hence $g^{-1}(V)$ and $g^{-1}(W)$ are disjoint clopen neighborhoods of $p$ and $q$ in $A$. Thus, $A$ is ultrahausdorff.

THEOREM 1. $S$ is ultraregular if and only if the topology on $S$ is the weak- $C^{*}(S, F)$ topology.

Proof. If $S$ is ultraregular, it has a clopen basis. Since $C^{*}(S, F)$ contains all characteristic functions of clopen sets, it follows that these basis sets are weak- $C^{*}\left(S, F^{\prime}\right)$ clopen as well. Hence the two topologies are identical.

To prove the converse, we apply Lemma 2, part (1), setting $A=$ $S, B=F$, and $G=C^{*}(S, F)$. Since $F$ is ultraregular, it follows that the weak- $C^{*}(S, F)$ topology on $S$ is ultraregular.

We will call $S$ mildly compact, or $M C$, if every clopen cover of $S$ has a finite subcover; mildly countably compact if every countable clopen cover has a finite subcover; and mildly Lindelof if every clopen cover has a countable subcover.

We mention several examples. The closed interval $[0,1]$, with the points $1,1 / 2,1 / 3, \cdots$ deleted, is mildly compact but not compact. A countably infinite set with discrete topology is mildly Lindelof, but not mildly countably compact. The space of all countable ordinals is mildly countably compact, but not mildly Lindelof [5].

LEMMA 3. $S$ is mildly countably compact if and only if every partition of $S$ into clopen sets is finite.

Proof. If $S$ is not mildly countably compact, it has a clopen cover $\left\{A_{i}: i=1,2,3, \cdots\right\}$ with no finite subcover. For each positive integer $n$, let $B_{n}=A_{n}-\bigcup\left\{A_{i}: 1 \leqq i<n\right\}$. Then the nonempty members of the family $\left\{B_{n}: n=1,2,3, \cdots\right\}$ form an infinite clopen partition of $S$. The proof of the converse is direct.

THEOREM 2. (1) An ultraregular, mildly compact space is compact. (2) An ultraregular, mildly Lindelof space is Lindelof.

Proof. If $S$ is ultraregular, it has a clopen basis. If $S$ is also mildly compact, then every covering of $S$ by members of this basis has a finite subcover. This last condition is sufficient for compactness [8]. The proof for mildly Lindelof spaces is similar.

The following diagrams of implications summarize some of our results. In these diagrams, COMP denotes "compact". 
Diagram 1. For all spaces,

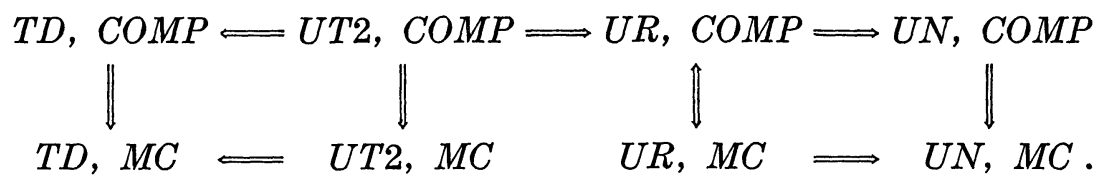

DIAGRAM 2. For Hausdorff spaces,

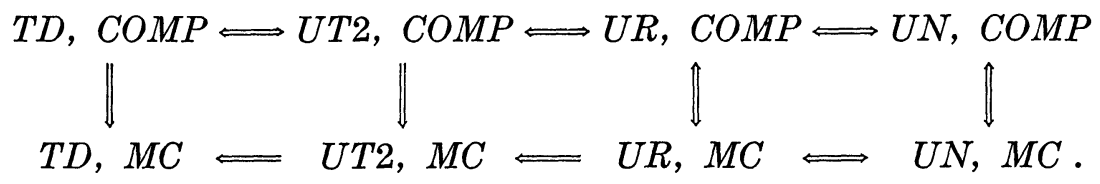

We note that all the implications of Diagram 2, except $T D$, $C O M P \Rightarrow U T 2, C O M P$, hold for $T_{1}$ spaces as well.

The quasicomponent quotient space of $S$, denoted $Q(S)$, will be the space of quasicomponents of $S$ with the quotient topology [9]. For each point $s$ of $S, Q(s)$ will denote the quasicomponent containing $s$. If $P$ is a clopen set in $S$, and hence a union of quasicomponents, then $Q(P)$ is clopen in $Q(S)$. If $S$ is ultraregular, we also have, using Lemma 1: If $P$ is open, then $Q(P)$ is open; and if $P$ is closed, then $Q(P)$ is closed. The following theorem is now obvious:

THEOREM 3. (1) $Q(S)$ is ultrahausdorff. The quotient mapping $Q: S \rightarrow Q(S)$ is a homeomorphism if and only if $S$ is ultrahausdorff.

(2) If $S$ is ultraregular, $Q(S)$ is ultraregular.

(3) If $S$ is compact, $Q(S)$ is compact.

(4) $Q(S)$ is mildly compact, or mildly countably compact, or mildly Lindelof, if and only if $S$ has the same property.

The ultraregular kernel of $S$, denoted $K(S)$, will be the space whose points are the points of $S$ and whose topology is generated by the clopen sets of $S$. It is obvious that:

Theorem 4. (1) The topology of $K(S)$ is the weak-C* $(S, F)$ topology (for any nonarchimedean field $F$ ).

(2) $K(S)$ is ultraregular. The topologies of $S$ and $K(S)$ are identical if and only if $S$ is ultraregular. in $S$.

(3) A subset of $S$ is clopen in $K(S)$ if and only if it is clopen

(4) $K(S)$ is ultrahausdorff if and only if $S$ is ultrahausdorff.

(5) If $S$ is compact, $K(S)$ is compact.

(6) $K(S)$ is mildly compact, or mildly countably compact, or mildly Lindelof, if and only if $S$ has the same property. 
We now show that the mappings $Q$ and $K$ commute.

TheOREM 5. $K(Q(S))$ and $Q(K(S))$ are identical topological spaces.

Proof. The points of both $K(Q(S))$ and $Q(K(S))$ are the quasicomponents of $S$. To show that the topologies are identical, we note that the following statements are equivalent:

$R$ is an open set in $K(Q(S))$.

$R$ is a union of clopen sets of $Q(S)$.

$Q^{-1}(R)$ is a union of clopen sets of $S$.

$Q^{-1}(R)$ is open in $K(S)$.

$R$ is open in $Q(K(S))$.

Henceforward, $Q K(S)$ will denote the topological space of Theorem 5. We note that this space is both ultrahausdorff and ultraregular.

A filter on $S$ with a base consisting of clopen sets will be called a cofilter, and a maximal cofilter will be called an ultracofilter. An arbitrary filter $H$ will be called fixed (after van der Put, [13]) if it has nonempty intersection; if $M$ is a cardinal number, $H$ will be called $M$-fixed if every intersection of $M$ members of $H$ is nonempty. We will say that $H$ recognizes a partition $\left\{A_{i}: i \in I\right\}$ of $S$ if one of the sets $A_{i}$ is in $H$. We note that a cofilter on $S$ is an ultracofilter if and only if it recognizes all finite partitions of $S$ into clopen sets. It is obvious that:

LEMma 4. If an ultracofilter on $S$ is $M$-fixed, for some infinite cardinal $M$, then it recognizes all clopen partitions of $S$ of cardinality $M$.

A partial converse to Lemma 4 is:

LEMMA 5. If a cofilter $H$ on $S$ recognizes all countable clopen partitions of $S$, then $H$ is countably fixed.

Proof. If $H$ is not countably fixed, then it contains a family $\left\{A_{i}: i=1,2,3, \cdots\right\}$ of clopen sets with empty intersection. For every positive integer $n$, let $B_{n}=\bigcap\left\{A_{i}: 1 \leqq i \leqq n\right\}$; then $\left\{B_{n}: n=1,2,3, \cdots\right\}$ is a family of clopen sets in $H$, ordered by exclusion, with empty intersection. Let $C_{1}=S-B_{1}$, and for $n>1$, let $C_{n}=B_{n-1}-B_{n}$. Then the family $\left\{C_{n}: n=1,2,3, \cdots\right\}$ forms a clopen partition of $S$, but none of these sets is in $H$. Hence $H$ does not recognize all clopen partitions of $S$.

Nonmeasurable cardinals [5] may be characterized as follows: every countably fixed ultrafilter on a set of nonmeasurable cardinality is fixed. It is known that the nonmeasurable cardinals include $\boldsymbol{\aleph}_{0}$, $\boldsymbol{N}_{1}, \boldsymbol{N}_{2}, \cdots$; they are closed under exponentiation, passage to a successor 
or to any smaller cardinal, and the supremum operation over a nonmeasurable index. The conjecture that all cardinals are nonmeasurable remains unproved; however, it is known that it can never be disproved.

Lemma 6. An ultracofilter $U$ on $S$ is countably fixed if and only if it recognizes all clopen partitions of $S$ of nonmeasurable cardinality.

Proof. If $U$ recognizes all clopen partitions of $S$ of nonmeasurable cardinality, then, by Lemma $5, U$ is countably fixed. Conversely, suppose $U$ is countably fixed and $\left\{A_{i}: i \in I\right\}$ is a clopen partition of $S$ of nonmeasurable cardinality. Then $U$ induces, via the quotient mapping, a countably fixed ultrafilter $U^{\prime}$ on the family $\left\{A_{i}: i \in I\right\}$. Since this family is of nonmeasurable cardinality, $U^{\prime}$ is fixed-that is, $U^{\prime}$ contains a singleton $\left\{A_{j}\right\}$. Hence $A_{j}$ is in $U$, so $U$ recognizes the partition $\left\{A_{i}: i \in I\right\}$.

Taking the dual versions of our compactness definitions, and using Lemma 6, we easily have the following lemmas:

LEMMA 7. $S$ is mildly compact if and only if every ultracofilter on $S$ is fixed.

LEMMA 8. The following are equivalent:

(1) $S$ is mildly countably compact.

(2) Every ultracofilter on $S$ is countably fixed.

(3) Every ultracofilter on $S$ recognizes all clopen partitions of $S$ of nonmeasurable cardinality.

Lemma 9. The following are equivalent:

(1) $S$ is mildly Lindelof.

(2) Every countably fixed ultracofilter on $S$ is fixed.

(3) Every ultracofilter on $S$ which recognizes all clopen partitions of $S$ of nonmeasurable cardinality is fixed.

2. The density of a nonarchimedean Banach space. Let $X$ be a nonarchimedean Banach space over $F$. We will assume that $|F| \subseteq\|X\| \leqq \mathrm{Cl}(|F|)$; i.e., (1) $X$ has a unit vector, and (2) if $F$ is discrete, then $\|X\|=|F|$.

A sphere $T(x, d)=\{y \in X:\|y-x\| \leqq d\}$ in $X$ will be called a closphere; a sphere $W(x, d)=\{y \in X:\|y-x\|<d\}$ will be called an osphere. $V(X)$ will denote the closphere and subring $T(0,1)$. All clospheres and ospheres are clopen sets; every point of a closphere or osphere is a center; and the clospheres, or ospheres, of any fixed radius form a partition of $X$ [1]. For any $x \in X, a \in F$, and $d>0$, we have: 


$$
a \cdot T(x, d)=T(a x,|a| \cdot d) \quad a \cdot W(x, d)=W(a x,|a| \cdot d) .
$$

THEOREM 6. If $X$ is locally compact, then every partition of a closphere in $X$ into clospheres of a fixed smaller radius is finite.

Proof. If $X$ is locally compact, it contains a compact sphere $K$. Since every other sphere in $X$ is homeomorphic, by a translation and scalar multiplication, to a clopen subset of $K$, it follows that every sphere in $X$ is compact. The theorem follows.

The remainder of this section is devoted to proving the companion theorem:

THEOREM 7. If $X$ is not locally compact, there exists an infinite cardinal $D(X)$ such that: if $d \in \mathrm{Cl}(|F|)$, then every partition of a closphere of radius $d$ in $X$ into clospheres of a fixed smaller radius is of cardinality $D(X)$.

For $0<d<1$, let $r(X, d)$ denote the cardinality of the partition of $V(X)$ into clospheres of radius $d$. Obviously, if $0<c<d<1$, then $r(X, c) \geqq r(X, d)$.

Lemma 10. If $0<c<1,0<d<1$, and $d \in|F|$, then $r(X, c d)=$ $r(X, c) \cdot r(X, d)$.

Proof. Choose $a \in F$ with $|a|=d$. Since $V(X)$ contains $r(X, c)$ distinct clospheres of radius $c$, it follows that $T(0, d)=a . \quad V(X)$ contains $r(X, c)$ distinct clospheres of radius $c d$. By translation, every closphere in $X$ of radius $d$ contains $r(X, c)$ distinct clospheres of radius $c d$; so $V(X)$, which contains $r(X, d)$ clospheres of radius $d$, must contain $r(X, c) \cdot r(X, d)$ clospheres of radius $c d$.

Corollary 11. If $0<d<1$ and $d \in|F|$, then $r\left(X, d^{n}\right)=r(X, d)^{n}$ for any positive integer $n$.

Lemma 12. If $X$ is not locally compact, and $0<d<1$, then $r(X, d)$ is infinite.

Proof. Shilkret [14] has shown that if $X$ is not locally compact, then it is not discrete or has infinite residue space. If $X$ is not discrete, then for some $d>0$ there is a family $\left\{x_{n}: n=1,2,3, \cdots\right\}$ in $X$ such that $d<\left\|x_{n}\right\|<1$ for all $n$ and $\left\|x_{n}\right\| \neq\left\|x_{m}\right\|$ for $n \neq m$. Hence the clospheres $\left\{T\left(x_{n}, d\right): n=1,2,3, \cdots\right\}$ are distinct in $V(X)$, so $r(X, d)$ is infinite.

If $X$ has infinite residue space, then the members of the residue 
space form a partition of $V(X)$ into ospheres of radius 1 . Since this partition is infinite, the equivalent or finer partition into clospheres of radius $d$ must also be infinite. Hence $r(X, d)$ is infinite.

Lemma 13. If $X$ is not locally compact, then the cardinal numbers $\{r(X, d): 0<d<1\}$ are all equal.

Proof. (1) Suppose $X$ and $F$ are not discrete, and $0<c<d<1$. Choose $e \in|F|$ and a positive integer $n$ such that $d<e<1$ and $e^{n}<c$. Applying Corollary 11 and Lemma 12, we have:

$$
r(X, d) \geqq r(X, e)=r(X, e)^{n}=r\left(X, e^{n}\right) \geqq r(X, c) \geqq r(X, d) .
$$

Hence $r(X, c)=r(X, d)$.

(2) Suppose $X$ and $F$ are discrete, with $\|X\|=|F|$, and $0<$ $d<1$. Let $e<1$ be a generator of the multiplicative group $|F-\{0\}|$, and let $n$ be the integer satisfying $e^{n} \leqq d<e^{n-1}$. Then every closphere of radius $d$ is a closphere of radius $e^{n}$; hence, by Corollary 11 and Lemma 12,

$$
r(X, d)=r\left(X, e^{n}\right)=r(X, e)^{n}=r(X, e) .
$$

For $X$ not locally compact, we now define $D(X)$, the density of $X$, to be $r(X, d)$ for any $d$ between 0 and 1 . For $X$ locally compact, it will be convenient to define $D(X)$ to be 1 .

Proof of Theorem 7. Suppose $X$ is not locally compact, $T(x, d)$ is a closphere in $X$ with $d \in \mathrm{Cl}(|F|)$, and $P$ is a partition of $T(x, d)$ into clospheres of radius $c<d$.

(1) If $d \in|F|$, then $T(x, d)$ is homeomorphic, by a translation and scalar multiplication, to $V(X)$; and this homeomorphism carries $P$, in a one-to-one fashion, onto the partition of $V(X)$ into clospheres of radius $c / d$. Hence, card $(P)=r(X, c / d)=D(X)$.

(2) If $d \in \mathrm{Cl}(|F|)$, then there exist $e, f \in|F|$ such that $c<e<$ $d<f$. Obviously, $T(x, e) \subseteq T(x, d) \subseteq T(x, f)$; and by part (1), $T(x, e)$ and $T(x, f)$ both contain precisely $D(X)$ clospheres of radius $c$. It follows that $T(x, d)$ contains $D(X)$ clospheres of radius $c$, so card $(P)=$ $D(X)$.

3. Maximal ideals of $C^{*}(S, F)$. Let $X$ be a commutative nonarchimedean Banach algebra over $F$, with identity $e$ of norm 1 .

Each quotient field $F_{M}=X / M$, for $M$ a maximal ideal of $X$, is both a field extending $F$ (identifying each $b \in F$ with $b \cdot e+M \in F_{M}$ ), and a normed algebra over $F$ whose quotient norm extends the valuation on $F[11]$. 
Each $x \in X$ gives rise to a function $x^{*}$ on $\mathfrak{M}$, the family of maximal ideals of $X$, given by $x^{*}(M)=x+M \in F_{M}(M \in \mathfrak{M})$. The family $X^{*}=$ $\left\{x^{*}: x \in X\right\}$, with the supremun norm, is a normed algebra over $F$.

After Shilkret [15], we let $\mathfrak{M}^{\prime}=\left\{M \in \mathfrak{M}: F_{M}=F\right\}$, and $X_{0}=$ $\left\{x \in X: x^{*}(M) \in F(M \in \mathfrak{M})\right\}$. On $\mathfrak{M}$, the Gelfand topology is the weak$X_{0}^{*}$ topology; on $\mathfrak{M}^{\prime}$, and all subsets of $\mathfrak{M}^{\prime}$, the strong Gelfand topology is the weak- $X^{*}$ topology, and the weak Gelfand topology is the weak$X_{0}^{*}$ topology.

THEOREM 8. (1) All Gelfand topologies are ultraregular. The strong Gelfand topology on $\mathfrak{M N}^{\prime}$ is ultrahausdorff.

Proof. We apply Lemma 2 , with $A=\mathfrak{M}^{\prime}$ or $\mathfrak{M}, B=F$, and $G=$ $X_{0}^{*}$ or $X^{*}$. Part (1) follows immediately; part (2) follows from the observation that $X^{*}$ separates points of $\mathfrak{M}^{\prime}$.

For the remainder of this paper, $X$ will be the algebra $C^{*}(S, F)$. $T(b, d)$ and $W(b, d)$ will denote clospheres and ospheres in $F . \quad \mathfrak{M}^{\prime \prime}$ will denote the family $\left\{M_{s}: s \in S\right\}$ of fixed maximal ideals of $X$, where $M_{s}=$ $\{x \in X: x(s)=0\}$. We note that $x^{*}\left(M_{s}\right)=x(s) \in F$ for all $x \in X$ and $M_{s} \in \mathfrak{M}^{\prime \prime} ;$ hence $\mathfrak{M}^{\prime \prime} \subseteq \mathfrak{M}^{\prime} \subseteq \mathfrak{M}$.

Theorem 9. For $s, t \in S: \quad M_{s}=M_{t}$ if and only if $Q(s)=Q(t)$.

Proof. If $M_{s} \neq M_{t}$, then $x(s)=0 \neq x(t)$ for some $x \in X$. Let $C$ be a clopen neighborhood of 0 in $F$ excluding $x(t)$; then $x^{-1}(C)$ is a clopen set in $S$ containing $s$ but not $t$. Hence $Q(s) \neq Q(t)$.

Conversely, if $Q(s) \neq Q(t)$, there is a clopen set $K$ in $S$ containing $s$ but not $t$. Since the characteristic function of $K$ belongs to $M_{t}$ but not $M_{s}$, we have $M_{s} \neq M_{t}$.

COROLlaRY 14. Each member of $X$ is constant on quasicomponents of $S$.

COROLLARY 15. There is a natural one-to-one correspondence between $Q K(S)$ and $\mathfrak{M N}^{\prime \prime}$, given by $Q(s) \rightarrow M_{s}(s \in S)$.

CoROLLARY 16. The natural mapping of $S$ onto $\mathfrak{M}^{\prime \prime}$ is a bijection if and only if $S$ is ultrahausdorff.

LEMMA 17. $X_{0}$ contains all characteristic functions in $X$.

Proof. If $x \in X$ is a characteristic function, then $x^{2}-x=0$. Hence, for all $M \in \mathfrak{M}, x^{*}(M)^{2}-x^{*}(M)=0$; so $x^{*}(M)=0$ or 1 . It follows that $x \in X_{0}$. 
THEOREM 10. The weak and strong Gelfand topologies on Mi" are identical. This topology is ultrahausdorff and ultraregular.

Proof. Let $\left(x^{*}\right)^{-1}(P)$ be a strong-Gelfand subbasic set in $\mathfrak{M}^{\prime \prime}$, where $P$ is an osphere in $F, x \in X$, and $x^{*}$ is regarded as a function on $\mathfrak{M}^{\prime \prime}$. Then

$\left(x^{*}\right)^{-1}(P)=\left\{M_{s}: x^{*}\left(M_{s}\right) \in P\right\}=\left\{M_{s}: x(s) \in P\right\}=\left\{M_{s}: s \in x^{-1}(P)\right\}$. Since $x^{-1}(P)$ is clopen in $S$, we have $x^{-1}(P)=y^{-1}(Q)$, where $y$ is the characteristic function of $x^{-1}(P), Q$ is a clopen set in $F$ containing 1 but not 0 , and $y^{*}$ is regarded as a function on $\mathfrak{M}^{\prime \prime}$. It follows that $\left(x^{*}\right)^{-1}(P)=$ $\left(y^{*}\right)^{-1}(Q)$, a weak-Gelfand open set. Hence the two Gelfand topologies on $\mathfrak{M}^{\prime \prime}$ are identical; by Theorem 8 , this topology is ultrahausdorff and ultraregular.

We can now speak of the Gelfand topology on $\mathfrak{M}^{\prime \prime}$ without ambiguity.

We now show that $C^{*}(S, F)$ is congruent to the algebra of bounded, continuous, $F$-valued functions on an ultrahausdorff, ultraregular space.

Theorem 11. $C^{*}(S, F)$ is congruent to $C^{*}\left(Q K(S), F^{\prime}\right)$.

Proof. Let $Q^{\prime}: C^{*}(Q K(S), F) \rightarrow C^{*}(S, F)$ be given by $Q^{\prime}\left(x^{\prime}\right)=x^{\prime} \circ Q$. Obviously, $Q^{\prime}$ is a ring homomorphism and an isometry. To show that $Q^{\prime}$ is onto $C^{*}(S, F)$, consider any $x \in C^{*}(S, F)$. Let $x^{\prime} \in C^{*}(Q K(S)$, $F)$ be given by $x^{\prime}(Q(s))=x(s)(s \in S)$. By Corollary 14, $x^{\prime}$ is welldefined; since $x$ is continuous on $S, x^{\prime}$ is continuous on $Q K(S)$; and obviously $Q^{\prime}\left(x^{\prime}\right)=x$.

Thus, in general, we can only hope to recover the structure of $Q K(S)$ from that of $X$. Only where $S$ is homeomorphic to $Q K(S)-$ i.e., where $S$ is ultraregular and $T_{1}$-can we hope to recover $S$ itself.

Using the facts that the topology on $Q K(S)$ is the weak- $C^{*}(Q K(S)$, $F$ ) topology, and the topology on $\mathfrak{M}^{\prime \prime}$ is the weak-C*(S,F)* topology, we easily have:

THEOREM 12. The natural bijection of $Q K(S)$ onto $\mathfrak{M}^{\prime \prime}$ is a homeomorphism.

CoRollary 18. The natural mapping of $S$ onto $\mathfrak{M}^{\prime \prime}$ is continuous; it is a homeomorphism if and only if $S$ is ultraregular and $T_{1}$.

We now establish a one-to-one correspondence between the closed ideals of $X$ and the cofilters on $S$.

For each $x \in X$, a smallset of $x$ will be a set

$$
\operatorname{Sm}(x, d)=\left\{s \in S:|x(s)|\langle d\}=x^{-1}(W(0, d)) \text { for some } d>0 .\right.
$$

Obviously, all smallsets are clopen. The zero-set $Z(x)$ will be the set 
$x^{-1}(0)$. For any clopen set $L$ in $S, C(L)$ will denote the characteristic function of $L$.

For any proper ideal $I$ of $X$, let $G^{\prime}(I)$ denote the family of all smallsets of members of $I$, and let $G^{\prime \prime}(I)$ denote the family of zerosets of characteristic functions of $I$.

Lemma 19. $G^{\prime}(I)=G^{\prime \prime}(I)$ for any ideal $I$ in $X$.

Proof. Let $N=S m(x, d) \in G^{\prime}(I)$. Let $y \in X$ be given by $y(s)=$ $0(s \in N), y(s)=x(s)^{-1}(s \notin N)$. Then $x y=C(S-N)$ is in $I$, so $N=$ $Z(x y)$ is in $G^{\prime \prime}(I)$. Thus, $G^{\prime}(I) \subseteq G^{\prime \prime}(I)$. The reverse inclusion is trivial.

We can now show that $G^{\prime}(I)$ generates a cofilter $G(I)$ on $S$. First, if $L, M \in G^{\prime}(I)$, then $C(S-L), C(S-M) \in I$; hence $C(S-L \cap M)=$ $C(S-L)+C(S-M)-C(S-L) \cdot C(S-M) \in I ; \quad$ so $\quad L \cap M \in G^{\prime}(I)$. Second, $\phi=Z(e) \notin G^{\prime}(I)$.

For any cofilter $G$ on $S$, let $I(G)$ denote the family of members of $X$ all of whose smallsets are in $G$.

Lemma 20. $I(G)$ is a closed ideal in $X$, for any cofilter $G$ on $S$.

Proof. If $x, y \in I(G)$, then for any $d>0, S m(x+y, d) \in G$, since $\operatorname{Sm}(x+y, d) \supseteqq \operatorname{Sm}(x, d) \cap \operatorname{Sm}(y, d) \in G$; hence $x+y \in I(G)$. If $x \in I(G)$ and $\mathrm{y} \in X$, then for any $d>0, \operatorname{Sm}(x y, d) \in G$, since $\operatorname{Sm}(x y, d) \supseteqq$ $\operatorname{Sm}\left(x, d\|y\|^{-1}\right) \in G$; hence $x y \in I(G)$, so $I(G)$ is an ideal. If $z \in \mathrm{Cl}(I(G))$, then for any $d>0,\|z-x\|<d$ for some $x \in I(G)$, so $\operatorname{Sm}(z, d)=$ $\operatorname{Sm}(x, d) \in G$; hence $z \in I(G)$, so $I(G)$ is closed.

Lemma 21. (1) $I(G(I))=\mathrm{Cl}(I)$ for any ideal $I$ in $X$. $G(I(G))=G$ for any cofilter $G$ on $S$.

Proof. (1) Let $x \in I(G(I))$. For any $d>0, S m(x, d) \in G(I)$; hence $S m(x, d)=z(C(L))$ for some $C(L) \in I$; so $x \cdot C(L) \in I$ and $\|x-x \cdot C(L)\|<$ d. Thus $x \in \mathrm{Cl}(I)$, so $I(G(I)) \subseteq \mathrm{Cl}(I)$. The reverse inclusion follows from the fact that $I(G(I))$ is a closed ideal containing $I$.

(2) Let $L \in G(I(G))$.' Then $L \supseteqq M$ for some $M \in G^{\prime}(I(G))$; hence $C(S-M) \in I(G)$, so $M=S m(C(S-M), 1 / 2) \in G$; so $L \in G$. Thus $G(I(G)) \cong G$. The reverse inclusion is trivial.

The following theorem is now obvious.

THEOREM 13. (1) There is a one-to-one correspondence between the proper closed ideals of $X$ and the cofilters on $S$, given by $I \rightarrow$ $G(I)$, with inverse $G \rightarrow I(G)$.

(2) This correspondence carries the maximal ideals of $X$ onto 
the ultracofilters on $S$, and the fixed maximal ideals onto the fixed ultracofilters.

THEOREM 14. $\mathfrak{M}^{\prime \prime}$ is Gelfand-dense in $\mathfrak{M}$, and weak-Gelfand dense in $\mathfrak{M}^{\prime}$.

Proof. Let $N \in \mathfrak{M}$, and let $V=\left\{M \in \mathfrak{M}:\left|x_{i}^{*}(M)\right|<d(1 \leqq i \leqq n)\right\}$ be a typical Gelfand-basic neighborhood of $N$, where $d>0$ and $x_{i} \in$ $X_{0} \cap N(1 \leqq i \leqq n)$. Then the smallsets $\left\{\operatorname{Sm}\left(x_{i}, d\right): 1 \leqq i \leqq n\right\}$ all belong to the ultracofilter $G(N)$; so the intersection of these smallsets is nonempty and contains a point $s$. Hence $\left|x_{i}^{*}\left(M_{s}\right)\right|=\left|x_{i}(s)\right|<d(1 \leqq$ $i \leqq n)$, so $M_{s} \in V$. Thus $N$ is in the Gelfand-closure of $\mathfrak{M}^{\prime \prime}$, and $\mathfrak{M}^{\prime \prime}$ is Gelfand-dense in $\mathfrak{M}$. The proof that $\mathfrak{M}^{\prime \prime}$ is weak-Gelfand dense in $\mathfrak{M}^{\prime}$ is similar.

CoRollary 22. The natural bijection of $Q K(S)$ onto $\mathfrak{M}^{\prime \prime}$ carries $Q K(S)$ onto a dense subspace of both $\mathfrak{M}$ and $\mathfrak{M}^{\prime}$.

From Lemma 7 and Theorem 13, we now have the key result:

THEOREM 15. $\mathfrak{M}^{\prime \prime}=\mathfrak{M}$ if and only if $S$ is mildly compact.

The following corollary will be of use later.

CoROLlary 23. Suppose $T$ is a mildly compact topological space, $I^{\prime}$ is an ideal in $C^{*}\left(T, F^{\prime}\right)$, and the members of $I^{\prime}$ do not all vanish at any point of $T$. Then $I^{\prime}=C^{*}(T, F)$.

Collecting results, we now nave the following theorem on the natural injection $B: Q(S) \rightarrow \mathfrak{M}$ and the mapping $B \circ Q: S \rightarrow \mathfrak{M}$.

THEOREM 16. (1) $B$ is a homeomorphism if and only if $S$ is mildly compact.

(2) $B \circ Q$ is a bijection if and only if $S$ is mildly compact and ultrahausdorff.

(3) $B \circ Q$ is a homeomorphism if and only if $S$ is compact and ultrahausdorff.

We note that, by Theorem $13, \mathfrak{M}=\{I(U)$ : $U$ an ultracofilter on $S\}$.

THEOREM 17. For any $x \in X,\|x\|=\left\|x^{*}(I(U))\right\|$ for some $I(U)$ in M; i.e., each member of $X$ realizes its norm on some maximal ideal.

Proof. Let $x \in X$. For $d>0$, let $K(d)=\{s \in S:|x(s)|>d\}$. The 
family $\{K(d): 0<d<\|x\|\}$ generates a cofilter on $S$, and is therefore contained in an ultracofilter $U$.

For $y \in I(U)$ and $0<d<\|x\|$, the sets $S m(y, d)$ and $K(d)$ are both in $U$; hence some $s \in S$ belongs to $S m(y, d) \cap K(d)$; so $|y(s)|<d$, $|x(s)|>d$, and $\|x-y\|>d$. Therefore, $\|x-y\| \geqq\|x\|$ for all $y \in I(U)$, so $\left\|x^{*}(I(U))\right\|=\inf \{\|x-y\|: y \in I(U)\} \geqq\|x\|$. The reverse inequality is trivial.

THEOREM 18. The following are equivalent:

(1) For any $x \in X,\|x\|=|x(s)|$ for some $s \in S$; i.e., each member of $X$ realizes its norm at some point of $S$.

(2) $F$ is discrete, or $S$ is mildly countably compact.

Proof. Suppose (2) is false. Then there is a clopen partition $\left\{A_{i}: i=1,2,3, \cdots\right\}$ of $S$, and a bounded sequence $\left\{b_{i}: i=1,2,3, \cdots\right\}$ in $F$ such that $\left\{\left|b_{i}\right|: i=1,2,3, \cdots\right\}$ is strictly increasing. Let $x \in X$ be given by $x(s)=b_{i}\left(x \in A_{i}, i \geqq 1\right)$. Then for any $s \in S,|x(s)|<\sup \left\{\left|b_{i}\right|: i=\right.$ $1,2,3, \cdots\}=\|x\|$. The proof of the converse is similar.

For $x \in X$, and $U$ an ultracofilter on $S, x(U)$ will denote the filter on $F$ generated by the family $\{x(T): T \in U\}$.

Lemma 24. If $x \in X, U$ is an ultracofilter on $S$, and $b \in F$, then $x^{*}(I(U))=b$ if and only if $x(U)$ converges to $b$.

Proof. Suppose $x^{*}(I(U))=b$. Then $(x-b \cdot e)^{*}(I(U))=0$, so $x-b \cdot e \in I(U)$. Hence, for any $d>0$,

$$
S m(x-b \cdot e, d)=\{s \in S:|x(s)-b|<d\}=x^{-1}(W(b, d))
$$

is in $U$, so $W(b, d)$ is in $x(U)$. Thus, every osphere containing $b$ is in $x(U)$, so $x(U)$ converges to $b$. The proof of the converse is similar.

Corollary 25. A maximal ideal $I(U)$ is in $\mathfrak{M}^{\prime}$ if and only if $x(U)$ converges for all $x \in X$.

THeOREM 19. $\mathfrak{M}^{\prime}=\mathfrak{M}$ (i.e., $F$ is the only quotient field of $X$ ) if and only if $F$ is locally compact or $S$ is mildly countably compact.

Proof. (1) Let $I(U) \in \mathfrak{M}$. For $x \in X$, and $d>0$, let $\left\{T\left(b_{i}, d\right): i \in I\right\}$ be a partition of the closphere $T(0,\|x\|)$ into clospheres of radius $d$. Then the nonempty members of the family $\left\{x^{-1}\left(T\left(b_{i}, d\right)\right): i \in I\right\}$ form a clopen partition of $S$. If $F$ is locally compact (in which case $I$ is finite) or $S$ is mildly countably compact, this partition must be finite; 
hence one set $x^{-1}\left(T\left(b_{j}, d\right)\right)$ is in $U$. Thus $T\left(b_{j}, d\right)$ is in $x(U)$, so $x(U)$ contains clospheres of arbitrarily small radius. Since $F$ is complete, $x(U)$ converges. By Corollary $25, I(U) \in \mathfrak{M}^{\prime}$; so $\mathfrak{M}^{\prime}=\mathfrak{M}$.

(2) Suppose $F$ is not locally compact and $S$ is not mildly countably compact. Then for some $d>0$, there is a bounded sequence $\left\{b_{i}: i=1,2,3, \cdots\right\}$ in $F$ such that $\left|b_{i}-b_{j}\right|>d$ for $i \neq j$; and there is a countable clopen partition $\left\{A_{i}: i=1,2,3, \cdots\right\}$ of $S$. The family $\left\{S-A_{i}: i=1,2,3, \cdots\right\}$ generates a cofilter on $S$, and is therefore contained in an ultracofilter $U$. Let $x \in X$ be given by $x(s)=b_{i}\left(s \in A_{i}\right.$, $i \geqq 1$ ). Then $x(U)$ contains no closphere of radius $d$, since it contains the complement of every such closphere. Hence $x(U)$ does not converge in $F$, so $I(U)$ is not in $\mathfrak{M}^{\prime}$. Thus $\mathfrak{M}^{\prime} \neq \mathfrak{M}$.

Lemma 26. A maximal ideal $I(U)$ belongs to $\mathfrak{M}^{\prime}$ if and only if $U$ recognizes all clopen partitions of $S$ of cardinality less than or equal to $D(F)$.

Proof. We may assume that $F$ is not locally compact, for otherwise the lemma is trivial. Suppose that $U$ recognizes all clopen partitions of $S$ of cardinality less than or equal to $D(F)$, and let $x \in$ $X$ and $0<d<\|x\|$. Let $\left\{T\left(b_{i}, d\right): i \in I\right\}$ be a partition of $T(0,\|x\|)$; then card $(I)=D(F)$. The nonempty members of the family $\left\{x^{-1}\left(T\left(b_{i}\right.\right.\right.$, $d)): i \in I\}$ form a clopen partition of $S$ of cardinality less than or equal to $D(F)$; hence $U$ contains a set $x^{-1}\left(T\left(b_{j}, d\right)\right.$ ). Thus, $T\left(b_{j}, d\right)$ is in $x(U) ; x(U)$ contains arbitrarily small spheres; $x(U)$ converges; and $I(U)$ is in $\mathfrak{M}^{\prime}$. The proof of the converse is similar to part (2) of the proof of Theorem 19.

Recalling Lemma 6, we have:

COROLlary 27. If $D(F)$ is an infinite, nonmeasurable cardinal, then $I(U)$ is in $\mathfrak{M}^{\prime}$ if and only if $U$ is countably fixed.

Using Theorems 13, 15, and 19, Lemmas 9 and 26, and Corollary 27 , we have the following theorems:

THEOREM 20. $\mathfrak{M}^{\prime \prime}=\mathfrak{M}^{\prime}$ if and only if every cofilter on $S$ which recognizes all clopen partitions of $S$ of cardinality less than or equal to $D(F)$ is fixed.

THEOREM 21. (1) For F locally compact: $\mathfrak{M}^{\prime \prime}=\mathfrak{M}^{\prime}$ if and only if $S$ is mildly compact.

(2) For F not locally compact: If $S$ is mildly Lindelof, then $\mathfrak{M}^{\prime \prime}=\mathfrak{M}^{\prime}$.

(3) For $F$ not locally compact and $D(F)$ nonmeasurable: $\mathfrak{M}^{\prime \prime}=$ 
$\mathfrak{M}^{\prime}$ if and only if $S$ is mildly Lindelof.

(4) For $S$ mildly countably compact: $\mathfrak{M N}^{\prime \prime}=\mathfrak{M}^{\prime}$ if and only if $S$ is mildly Lindelof.

The question of whether $\mathfrak{M}^{\prime \prime}=\mathfrak{M}^{\prime}$ always implies that $S$ is mildly Lindelof remains open.

COROLlary 28. Suppose $F$ is not locally compact. Then:

(1) If $S$ is $T_{1}$, ultraregular, and mildly Lindelof, then $B \circ Q$ is a homeomorphism of $S$ onto $\mathfrak{M}^{\prime}$.

(2) If $D(F)$ is nonmeasurable, and $B \circ Q$ carries $S$ homeomorphically onto $\mathfrak{M}^{\prime}$, then $S$ is $T_{1}$, ultraregular, and mildly Lindelof.

4. Stone-Weierstrass properties. We will say that $X$ has the stong Stone-Weierstrass property if every closed subalgebra which separates quasicomponents of $S$ is either $X$ itself or a fixed maximal ideal; and that $X$ has the weak Stone-Weierstrass property if the only closed subalgebra which separates quasicomponents and contains the constants is $X$ itself. This section is devoted to proving the following two theorems:

THEOREM 22. $X$ has the strong Stone-Weierstrass property if and only if $S$ is mildly compact.

THEOREM 23. If $X$ has the weak Stone-Weierstrass property, then $S$ is mildly countably compact.

We begin with a lemma of Kaplansky [7].

Lemma 29. If $D$ is a compact set in $F$, and $0 \neq a \in F$, then there is a polynomial $p(t)$ over $F$, without constant term, such that $p(a)=$ 1 and $|p(b)| \leqq 1(b \in D)$.

Proof. We may assume that $a \in D$, for otherwise we can replace $D$ with $D \cup\{a\}$. Let $d=|a|^{2} /|D|$, where $|D|=\sup \{|b|: b \in D\}$. Then $d \leqq|a|$.

The set $D-T(0,|\alpha|)$ is closed in $D$, hence compact; hence it has a finite partition $\left\{T\left(b_{i}, d\right) \cap D: 1 \leqq i \leqq n\right\}$ into clospheres of radius $d$. We may assume that $\left|b_{1}\right| \leqq\left|b_{2}\right| \leqq \cdots \leqq\left|b_{n}\right|$. We $\operatorname{set} k(i)=2^{i-1}(1 \leqq i \leqq n)$ and

$$
p(t)=1-(1-t / a) \cdot \prod_{i=1}^{n}\left(1-t / b_{i}\right)^{k(i)} .
$$

By straightforward computation, the lemma follows. 
Proof of Theorem 22. (1) If $S$ is not mildly compact, then, by Theorem 15, $X$ has a nonfixed maximal ideal $I(U)$. If $Q$ and $R$ are distinct quasicomponents of $S$, then some clopen set $A \subseteq S$ contains $Q$ but not $R$; either $A$ or $S-A$ is in $U$; and hence a characteristic function in $I(U)$ separates $Q$ and $R$. Thus, $I(U)$ is a closed subalgebra of $X$ which separates quasicomponents. Since $I(U)$ is neither $X$ itself nor a fixed maximal ideal, $X$ does not have the strong Stone-Weierstrass property.

(2) Suppose $S$ is mildly compact, and $Y$ is a closed subalgebra of $X$ which separates quasicomponents. We will prove that if $Y$ is contained in some fixed maximal ideal $M_{s}$, then $Y=M_{s}$; a similar proof shows that if $Y$ is not contained in any fixed ideal, then $Y=X$.

We therefore assume that $Y \subseteq M_{s}$ for some $s \in S$. First, we contend that if $u, v \in S$ and $Q(s) \neq Q(u) \neq Q(v)$, then some $x_{v} \in Y$ satisfies: $x_{v}(u)=1, x_{v}(v)=0,\left\|x_{v}\right\|=1$.

To prove this, we note that some $y_{1} \in Y$ separates $u$ and $v$; and some $y_{2} \in Y$ does not vanish at $u$. Let $y=y_{1} y_{2}-y_{1}(v) \cdot y_{2}$; then $y \in Y$, $y(u) \neq 0$, and $y(v)=0$. Let $a=y(u)$ and $D=y(S)$; then $D$ is mildly compact, hence compact. Let $p(t)$ be the resulting polynomial of Lemma 29, and let $x_{v}=p(y)$.

Second, we contend that if $V$ is a clopen set in $S$ containing $s, u$ is a point of $S$ outside $V$, and $d>0$, then some $x \in Y$ satisfies: $x(u)=$ $1,|x(v)| \leqq d(v \in V)$, and $\|x\|=1$.

To prove this, we note that $V$ is mildly compact. For each $v \in V$, some $x_{v} \in Y$ satisfies: $x_{v}(u)=1, x_{v}(v)=0$, and $\left\|x_{v}\right\|=1$. For $v \in V$, let $W_{v}=\left\{w \in V:\left|x_{v}(w)\right|<d\right\}$; then the family $\left\{W_{v}: v \in V\right\}$ is a clopen cover of $V$ and has a finite subcover $\left\{W_{v(1)}, \cdots, W_{v(n)}\right\}$. Let $x=$ $x_{v(1)} \cdot x_{v(2)} \cdots x_{v(n)}$.

Third, we contend that if $W$ is a clopen set in $S$ not containing $s$, then the characteristic function $C(W)$ is in $Y$.

To prove this, we note that if $0<d<1$ and $u \in W$, then some $x_{u} \in Y$ satisfies: $x_{u}(u)=1,\left|x_{u}(v)\right|<d(v \in S-W)$, and $\left\|x_{u}\right\|=1$. Each set $W_{u}=\left\{w \in W:\left|x_{u}(w)-1\right|<d\right\}$ is clopen in $W$; hence the family $\left\{W_{u}: \mathrm{u} \in W\right\}$ is a clopen cover of $W$; and since $W$ is mildly compact, a finite subfamily $\left\{W_{u(1)}, \cdots, W_{u(n)}\right\}$ covers $W$. Let

$$
x=e-\left(e-x_{u(1)}\right) \cdots\left(e-x_{u(n)}\right) \text {; }
$$

then $x \in Y$ and $\|x-C(W)\| \leqq d$. Since $Y$ is closed, $C(W) \in Y$.

Finally, we show that $Y=M_{s}$. Let $x \in M_{s}$; for any $d>0$, the preimages, under $x$, of the clospheres of radius $d$ in $F$ form a clopen partition of $S$. Since $S$ is mildly compact, this partition is finite: $S=\bigcup\left\{W_{i}: 1 \leqq i \leqq n\right\}$, where each $W_{i}=x^{-1}\left(T\left(a_{i}, d\right)\right)=\{u \in S: \mid x(u)-$ $\left.a_{i} \mid \leqq d\right\}$ for some $a_{i} \in F$. We may assume that $a_{1}=0$; i.e., that $s \in W_{1}$. Let $y=a_{1} C\left(W_{1}\right) \cdots a_{n} C\left(W_{n}\right)$; then $y \in Y$ and $\|x-y\| \leqq d$. Since $Y$ 
is closed, $x \in Y$.

Proof of Theorem 23. Suppose $S$ is not mildly countably compact. Then $S$ has an infinite clopen partition $\left\{T_{i}: i \in I\right\}$. Let $Y$ be the closed ideal in $X$ generated by the characteristic functions of these sets; and let $Z$ be the subalgebra $Y+F \cdot e$ of $X$. Then $Z$ is a closed subalgebra of $X$ which separates quasicomponents and contains the constants. However, every member of $Z$ must take values arbitrarily close to some constant on all but a finite number of the sets $\left\{T_{i}: i \in I\right\}$; so $Z$ is not equal to $X$ itself. Hence $X$ does not have the weak Stone-Weierstrass property.

We note that the question of whether the weak Stone-Weierstrass property is equivalent to mild compactness, or to mild countable compactness, or to some intermediate property, remains unresolved.

\section{REFERENCES}

1. N. Bourbaki, General Topology (2 vol.), Addison-Wesley, Reading, Mass., 1966.

2. D. G. Cantor, On the Stone-Weierstrass approximation theorem for valued fields, Pacific J. Math., 21 (1967), 473-478.

3. P. R. Chernoff, R. A. Rasala, and W. C. Waterhouse, The Stone-Weierstrass theorem for valuable fields, Pacific J. Math., 27 (1968), 233-240.

4. R. L. Ellis, A nonarchimedean analog of the Tietze-Urysohn extension theorem, Indag. Math., 70 (1967), 332-333.

5. L. Gillman and M. Jerison, Rings of Continuous Functions, Van Nostrand, New York, 1960.

6. J. G. Hocking and G. S. Young, Topology, Addison-Wesley, Reading, Mass., 1961.

7. I. Kaplansky, The Weierstrass theorem in fields with valuation, Proc. Amer. Math. Soc., 1 (1950), 356-357.

8. J. L. Kelley, General Topology, Van Nostrand, New York, 1955.

9. K. Kuratowski, Topology (2 vol.), Academic Press, New York, 1966-1968.

10. A. F. Monna, Analyse Non-archimedienne, Springer-Verlag, Berlin, 1970.

11. L. Narici, On nonarchimedean Benach algebras, Arch. Math., 19 (1968), 428-435.

12. L. Narici, E. Beckenstein, and G. Bachman, Functional Analysis and Valuation Theory, Dekker, New York, 1971.

13. M. van der Put, Algebres de fonctions continues p-adiques, Indag. Math., 30 (1968), 401-420.

14. N. Shilkret, Non-archimedean Banach algebras, Doctoral dissertation, Polytechnic Institute of Brooklyn, 1968.

15. — Nonarchimedean Gelfand theory, Pacific J. Math., 32 (1970), 541-550.

Received August 4, 1972. Taken in part from the dissertation submitted to the Faculty of the Polytechnic Institute of Brooklyn in partial fulfillment of the requirements for the degree of Doctor of Philosophy, 1970, under the guidance of Prof. George Bachman.

Sponsored in part by a grant from the City University of New York Faculty Research Program. 



\section{PACIFIC JOURNAL OF MATHEMATICS}

\section{EDITORS}

RICHARD ARENS (Managing Editor) University of California

Los Angeles, California 90024

R. A. BeaUmont

University of Washington Seattle, Washington 98105
J. DUGUNDJI*

Department of Mathematics University of Southern California Los Angeles, California 90007

D. Gilbarg and J. Milgram Stanford University

Stanford, California 94305

\section{ASSOCIATE EDITORS}

E. F. BECKENBACH

B. H. NeUmanN

F. WOLF

K. YoSHIDA

\section{SUPPORTING INSTITUTIONS}

\section{UNIVERSITY OF BRITISH COLUMBIA CALIFORNIA INSTITUTE OF TECHNOLOGY UNIVERSITY OF CA.LIFORNIA MONTANA STATE UNIVERSITY UNIVERSITY OF NEVADA NEW MEXICO STATE UNIVERSITY OREGON STATE UNIVERSITY UNIVERSITY OF OREGON OSAKA UNIVERSITY}

UNIVERSITY OF SOUTHERN CALIFORNIA STANFORD UNIVERSITY UNIVERSITY OF TOKYO UNIVERSITY OF UTAH WASHINGTON STATE UNIVERSITY UNIVERSITY OF WASHINGTON AMERICAN MATHEMATICAL SOCIETY NAVAL WEAPONS CENTER

* C. R. DePrima California Institute of Technology, Pasadena, CA 91109, will replace J. Dugundji until August 1974. 


\section{Pacific Journal of Mathematics}

\section{Vol. 50, No. $1 \quad$ September, 1974}

Gail Atneosen, Sierpinski curves in finite 2-complexes.............. 1

Bruce Alan Barnes, Representations of $B^{*}$-algebras on Banach spaces .... 7

George Benke, On the hypergroup structure of central $\Lambda(p)$ sets ....... 19

Carlos R. Borges, Absolute extensor spaces: a correction and an

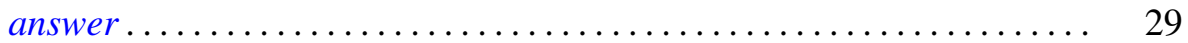

Tim G. Brook, Local limits and tripleability .................. 31

Philip Throop Church and James Timourian, Real analytic open maps .... 37

Timothy V. Fossum, The center of a simple algebra ............... 43

Richard Freiman, Homeomorphisms of long circles without periodic

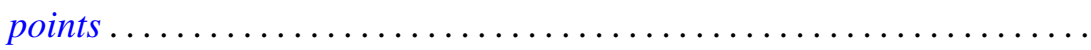

B. E. Fullbright, Intersectional properties of certain families of compact

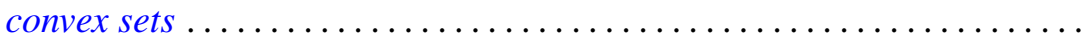

Harvey Charles Greenwald, Lipschitz spaces on the surface of the unit

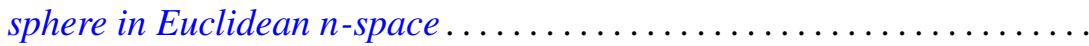

Herbert Paul Halpern, Open projections and Borel structures for

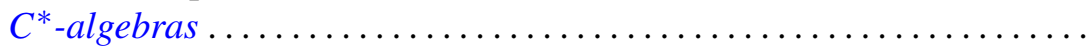

Frederic Timothy Howard, The numer of multinomial coefficients divisible

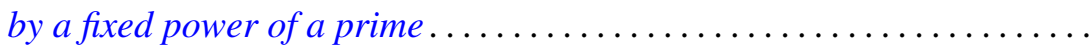

Lawrence Stanislaus Husch, Jr. and Ping-Fun Lam, Homeomorphisms of manifolds with zero-dimensional sets of nonwandering points........ 109

Joseph Edmund Kist, Two characterizations of commutative Baer rings ...

Lynn McLinden, An extension of Fenchel's duality theorem to saddle functions and dual minimax problems ................

Leo Sario and Cecilia Wang, Counterexamples in the biharmonic classification of Riemannian 2-manifolds...

Saharon Shelah, The Hanf number of omitting complete types ...

Richard Staum, The algebra of bounded continuous functions into a

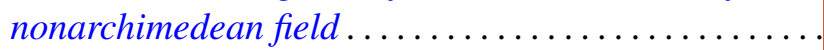

James DeWitt Stein, Some aspects of automatic continuity ..

Tommy Kay Teague, On the Engel margin

John Griggs Thompson, Nonsolvable finite groups all of whose local subgroups are solvable, $V \ldots \ldots \ldots \ldots \ldots \ldots \ldots \ldots$

Kung-Wei Yang, Isomorphisms of group extensions 